\title{
A biological target for antiplatelet therapy: the prostaglandin $\mathrm{E}_{2}$ receptor $\mathrm{EP}_{4}$
}

\author{
Sonia Philipose, Martina Ofner, Ákos Heinemann, Rufina Schuligoi \\ From 16th Scientific Symposium of the Austrian Pharmacological Society (APHAR) \\ Vienna, Austria. 25-27 November 2010
}

\begin{abstract}
Background
Acute myocardial infarction is one of the leading causes of death in the world which is caused by coronary artery thrombosis. Platelets play a central role in cardiovascular thrombosis. Platelet aggregation caused due to a ruptured artherosclerotic plaque could eventually lead to vascular occlusion. Another important component of vascular diseases is inflammation. During inflammation, prostaglandins (PG) like $\mathrm{PGI}_{2}, \mathrm{PGE}_{2}$ and $\mathrm{PGD}_{2}$ are released which are also involved in thrombosis. Lower concentrations of $\mathrm{PGE}_{2}$ enhance platelet aggregation whereas higher concentrations inhibit aggregation. $\mathrm{PGE}_{2}$ acts via 4 receptors: $\mathrm{EP}_{1}, \mathrm{EP}_{2}, \mathrm{EP}_{3}$ and $\mathrm{EP}_{4}\left(\mathrm{G}_{\mathrm{s}}\right.$ signalling). The role of the $\mathrm{EP}_{3}$ receptor in enhancing platelet activation and aggregation has been looked at in detail but the role of the $\mathrm{EP}_{4}$ receptor is largely unknown. We were interested in how this receptor modulates platelet aggregation and what are the signalling mechanisms involved in this process.
\end{abstract}

\section{Methods}

Platelet aggregation assays were performed ex vivo using a platelet aggregation analyser (Aggregometer II). Blood from healthy human donors was used to obtain plateletrich plasma. Aggregation was induced using ADP or collagen. Different agonists and antagonists were added to investigate their effects on platelet aggregation. $\mathrm{Ca}^{2+}$ flux changes caused by addition of agonists were also examined using a fluorescent $\mathrm{Ca}^{2+}$ dye (Fluo-3) by flow cytometry. Expression of the $\mathrm{EP}_{4}$ receptor on the surface of platelets was established using indirect flow cytometry whereas expression of CD62P, PAC1 and CD41 was examined using direct flow cytometry. In vitro thrombus formation was assessed by flowing whole blood on collagen-coated Cellix biochips at -30 dyne $/ \mathrm{cm}^{2}$ using the Mirus nanopump.

\section{Results}

We observed that human platelets express $\mathrm{EP}_{4}$ receptors. A selective $\mathrm{EP}_{4}$ agonist potently inhibited the platelet aggregation as induced by ADP or collagen. This effect could be completely reversed by using an $\mathrm{EP}_{4}$ antagonist, but not by $\mathrm{PGI}_{2}, \mathrm{PGD}_{2} \mathrm{TXA}_{2}$ receptor antagonists. Moreover, an $\mathrm{EP}_{4}$ antagonist enhanced the $\mathrm{PGE}_{2}$-induced stimulation of platelet aggregation, indicating a potent anti-aggregatory activity of the $\mathrm{EP}_{4}$ receptors. Interestingly, the inhibitory effect of the $\mathrm{EP}_{4}$ agonist was brought about by protein kinase $\mathrm{C}$ but not adenylyl cyclase, accompanied by attenuated $\mathrm{Ca}^{2+}$ flux, decreased activation of glycoprotein IIb/IIIa and downregulation of $\mathrm{P}$-selectin. Most importantly, in vitro thrombus formation was effectively reduced by the $\mathrm{EP}_{4}$ agonist and this effect was reversed using the $\mathrm{EP}_{4}$ antagonist.

\section{Conclusions}

These findings indicate that the $\mathrm{EP}_{4}$ receptor is a potential biological drug target in anti-platelet therapy.

Published: 16 November 2010

doi:10.1186/1471-2210-10-S1-A17

Cite this article as: Philipose et al:: A biological target for antiplatelet therapy: the prostaglandin $\mathrm{E}_{2}$ receptor $\mathrm{EP}_{4}$. BMC Pharmacology 2010 10(Suppl 1):A17.

\footnotetext{
* Correspondence: rufina.schuligoi@medunigraz.at 